\title{
Experimental Evaluation of a High Fineness Ratio Body With Drag Brakes
}

\author{
Corey J. Florendo*, Thomas R. Yechout**, Stefan Siegel***, and Russell M. Cummings** \\ Department of Aeronautics, United States Air Force Academy \\ and \\ Joseph Kealos**** \\ Textron, Inc., Wilmington, MA
}

This paper discusses the design evolution of a high fineness ratio body with drag brakes focusing on the experimental testing that directly contributed to definition of the final design configuration. A parallel computational fluid dynamics (CFD) effort was accomplished which nicely complimented the experimental program and which is the subject of a separate paper. The flight vehicle described in this paper was called the Advanced Remote Ground Unattended Sensor, or ARGUS, and was intended to be deployed from an aircraft, conduct free flight where it would transition from approximately a level attitude to a vertical attitude, and then impact the ground. The United States Air Force Academy provided critical aerodynamic support for the design and development of this vehicle through wind tunnel research. Initial flight tests had shown that the ARGUS predecessor (the Steel Eagle II) lacked sufficient aerodynamic stability to impact the ground in a near-vertical attitude due to a steady-state roll/yaw oscillation referred to as "coning." Wind-tunnel and CFD evaluations began in the Fall of 2003 to understand/correct this problem and to contribute to evolution and definition of the ARGUS design. During this initial effort, four problems were identified. First, the lift characteristics of the ARGUS were found to, upon release, potentially cause the ARGUS to rise back toward the carrier aircraft and create a hazard. Second, yawing moment excursions were identified that would perturb the ARGUS from a trimmed condition, and third, after such a perturbation, the stabilizing pitching moment of the ARGUS was found to be very limited in restoring the trimmed condition. Finally, the terminal velocity of the initial ARGUS design was found to be approximately 15\% lower than the desired terminal velocity of $265 \mathrm{ft} / \mathrm{sec}$. The yaw and pitching moment characteristics were identified as the probable cause of the coning experienced during the preliminary flight tests. To correct the coning problem, a perforated drag brake design (as an alternative to the initial solid drag brake design) was suggested to reduce asymmetric vortex shedding that was predicted to be occurring behind the solid drag brakes of the initial ARGUS design. The perforated drag brake design was found to significantly improve the performance of ARGUS and have a positive effect on the four problems identified, giving the ARGUS desirable aerodynamic characteristics. A new investigation was then performed in an attempt to optimize the perforation pattern on the drag brakes. Five perforation patterns were evaluated, including the "baseline" pattern from the initial testing. This testing led to selection of the baseline pattern for the final design. Results from flight test confirmed that this design mitigated the coning effect that was previously seen and would result in satisfactory ARGUS performance. During this effort, the ARGUS increased in size, so the drag data from previous testing was used to correctly size the drag brakes so that a desired terminal velocity of approximately $265 \mathrm{ft} / \mathrm{sec}$ could be achieved. A final wind tunnel investigation was conducted to establish baseline aerodynamic data for the final ARGUS design and to investigate the aerodynamic effects of the addition of a release lanyard system to the ARGUS. The lanyard system was not found to diminish the aerodynamic characteristics of the ARGUS, and the final ARGUS design was found to have aerodynamic characteristics suitable for its mission. The overall ARGUS design effort through all phases of testing was led by Textron, Inc. under contract to the Air Force Electronic Systems Center at Hanscom AFB. This paper presents the 18-month ARGUS design evolution from an aerodynamic view and the crucial data and analysis that led to definition of the final successful design.

\footnotetext{
* Student Member, AIAA

** Associate Fellow, AIAA

**** Senior Member, AIAA

****Member, AIAA
} 


\section{Nomenclature}

$\begin{array}{ll}\alpha & =\text { angle of attack } \\ \rho & =\text { density } \\ B_{i} & =\text { test instrument overall bias error } \\ C_{D} & =\text { drag coefficient } \\ C_{L} & =\text { lift coefficient } \\ C_{M} & =\text { pitching moment coefficient } \\ C_{N} & =\text { yawing moment coefficient } \\ D & =\text { drag } \\ M & =\text { Mach number } \\ P_{i} & =\text { test instrument overall precision error } \\ S & =\text { reference area used in coefficient reduction } \\ U_{i} & =\text { test instrument overall uncertainty } \\ V_{T} & =\text { terminal velocity } \\ W & =\text { weight }\end{array}$

\section{Background}

In the Fall of 2003, the United States Air Force Academy Department of Aeronautics was tasked to provide aerodynamic support for the development of the Advanced Remote Ground Unattended Sensor (ARGUS). This support included wind tunnel and computational-fluid-dynamics (CFD) analysis. The ARGUS was a proposed system planned for the US Air Force. After being dropped from a carrier aircraft, the ARGUS was designed to deploy drag brakes to slow it to a pre-determined terminal velocity, and then penetrate the ground. It was crucial that the ARGUS impact the ground in a near-vertical attitude to meet ground penetration and structural requirements. During initial flight tests of the ARGUS predecessor (the Steel Eagle II), "coning" tendencies were experienced in flight, meaning that after being dropped from an aircraft, the Steel Eagle II would not transition to stable, trimmed flight at zero degrees angle of attack. Instead, it would oscillate in a circular motion about its center of gravity, and thus would not impact the ground in a vertical attitude. A focus of this effort was to understand the cause of coning and develop a design approach which would mitigate it. In addition, overall aerodynamic characteristics had to be defined and the drag of the new ARGUS design had to be matched to the weight to achieve the desired terminal velocity for penetration.

\section{Objectives}

The overall objective of this effort was to define the aerodynamic and stability characteristics of the ARGUS and contribute to the evolution of an optimal design.

\section{Experimental Methods}

For wind tunnel testing, a $61.5 \%$ scale model was fabricated of the ARGUS. The initial full-scale ARGUS dimensions are shown in Fig 1. ARGUS is composed of three primary sections: a forebody, an aft body, which is distinguished by a larger diameter than the forebody, and four drag brakes which surround the aft body. The area of the aft body behind the drag brakes is commonly referred to as the "tail cone" of the ARGUS, and this area was a primary focus of the aerodynamic testing of the ARGUS. Fig. 2 shows the aft body of the ARGUS model used in testing. The $61.5 \%$ ARGUS model was mounted on an ABLE internal force balance, which was

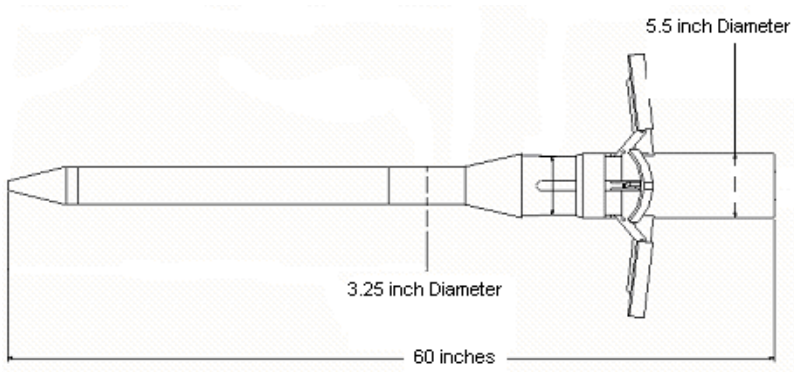

Figure 1. ARGUS Initial Design (Full-Scale).

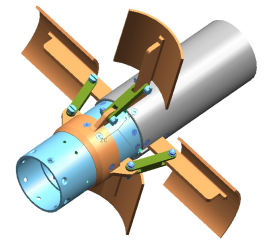

Figure 2. ARGUS Aft Body Used For Phase ${ }_{2} \mathbf{I}$ Testing With Solid Drag Brakes.

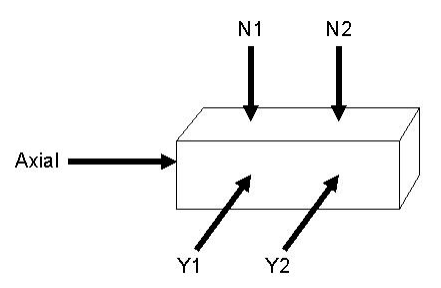

Figure 3. ABLE Force Balance Diagram.

American Institute of Aeronautics and Astronautics 


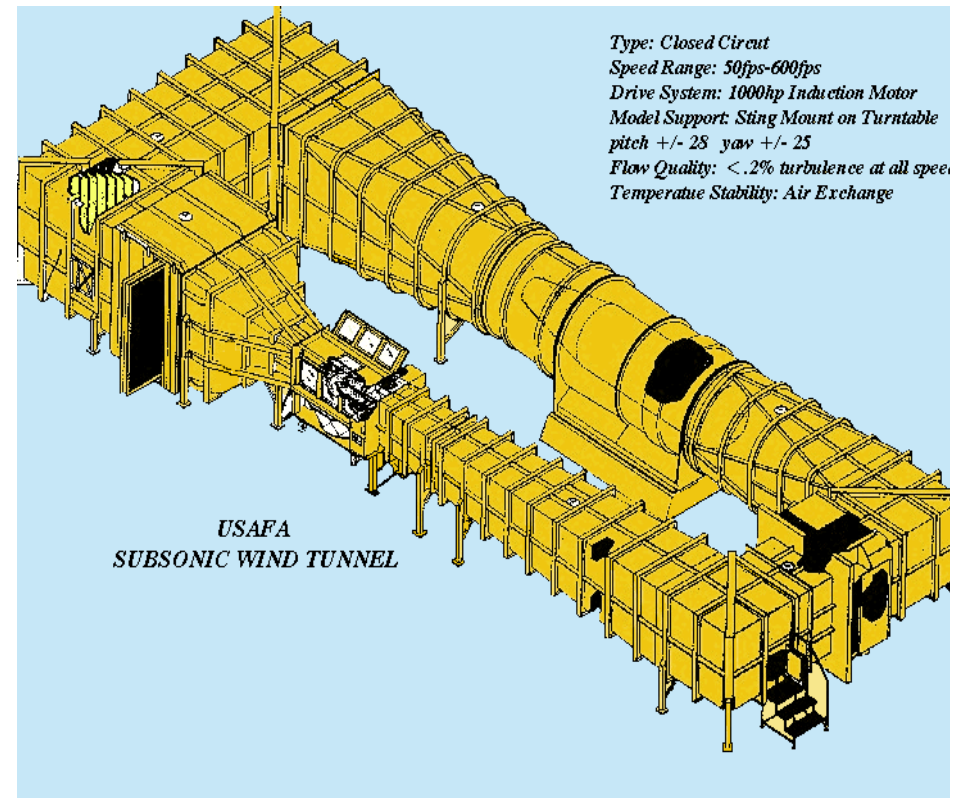

Figure 4. USAFA Subsonic Wind Tunnel Diagram. then mounted on a sting in the test section of the wind tunnel. Five measurements were taken from this force balance, as is shown in Fig. (3). The $61.5 \%$ scale was chosen to keep wind-tunnel blockage in the test section below $5 \%$ based on frontal area. The actual blockage at the highest angle of attack for most testing was under $3 \%$. Data was gathered in the sub-sonic wind tunnel at USAFA, which is presented in Fig (4). This wind tunnel had a 3' by 3' test section and a maximum Mach number of 0.6. All testing was accomplished between Mach 0.2 and Mach 0.5. Mach 0.5 was chosen due to the fact that the wind tunnel was approaching its operating power limit with the high drag of the ARGUS model, and Mach 0.2 was chosen as it is approximately the desired terminal velocity of the ARGUS. The angle of attack range examined was $-4^{\circ}$ to $20^{\circ}$ for most tests. Due to the fact that the wind tunnel was a

closed-loop, single-return tunnel, a small amount of flow angularity was present in the test section. To counter this, a yaw (or beta) offset of $0.3^{\circ}$ was utilized in most tests. From the differences in forces recorded on the Axial, N1, and $\mathrm{N} 2$ components, the drag, lift, and pitching moment coefficients could be determined at any angle-of-attack of the model. Similarly, the differences in Y1 and Y2 allowed for the calculation of the yawing moment coefficient. Data was acquired and reduced on an HP3853 Data Acquisition System using software developed at the United States Air Force Academy. At each test point, data samples were taken at $100 \mathrm{~Hz}$ for 2 seconds for all phases of testing, and those data samples were averaged to produce time-averaged data. The final series of tests (Phase III) investigated the time history of the data to observe any unsteady phenomenon.

There were four primary aerodynamic criteria used throughout the ARGUS investigation. The lift was examined to ensure a positive lift-curve slope was present, especially at low angles of attack (where the ARGUS will be released from the carrier aircraft and therefore pose the greatest threat to that aircraft). This positive lift-curve slope ensures that as the ARGUS decreases its angle of attack, its lift also decreases. If a negative lift-curve slope were present, the ARGUS would increase lift as its angle of attack decreased after release, possibly moving it back in the direction of the carrier aircraft. The second criterion was the pitching moment or the longitudinal static stability. As the angle of attack increased from trim, longitudinal stability required that the ARGUS experience an inherent pitching moment back to the trim condition. Higher stability was indicated by a steeper negative slope of pitching moment as a function of angle of attack. In the third criteria, the yawing moment was examined to ensure yaw excursions were minimized as variations were made in angle of attack. Minimal yawing moment excursions, combined with longitudinal static stability, were predicted to contribute to the mitigation of the coning tendency discussed earlier. Finally, the drag of each ARGUS configuration was examined to determine how closely each design matched the target terminal velocity of $265 \mathrm{ft} / \mathrm{sec}$. Each of the 4 primary aerodynamic characteristics were put into coefficient form. The lift coefficient $\left(\mathrm{C}_{\mathrm{L}}\right)$, drag coefficient, $\left(C_{D}\right)$, pitching moment coefficient $\left(C_{M}\right)$, and yawing moment coefficient $\left(\mathrm{C}_{\mathrm{N}}\right)$ were calculated using the normalization

Table 1. ARGUS Coefficient Reference Dimensions For 61.5\% Scale Model.

\begin{tabular}{|c|c|c|}
\hline Testing Period & $\begin{array}{c}\text { Reference } \\
\text { Length }\end{array}$ & $\begin{array}{c}\text { Reference } \\
\text { Area }\end{array}$ \\
\hline Phase I & $\mathbf{2 . 0 0}$ in & $\mathbf{3 . 1 4}^{\text {in }}$ \\
\hline Phase II and III & $\mathbf{2 . 1 5 4}$ in & $\mathbf{3 . 6 4 2}^{2}{ }^{2}$ \\
\hline
\end{tabular}
values presented in Table (1).

The terminal velocity of the ARGUS was calculated using Eqn. 1, where D is the drag, $\rho$ is the density of the air (assumed to be at sea-level on a standard day), $\mathrm{V}_{\mathrm{T}}$ is the terminal velocity, $\mathrm{S}$ is the reference area used in coefficient reduction, and $\mathrm{W}$ is the weight of the ARGUS.

$$
D=C_{D} \frac{1}{2} \rho V_{T}^{2} S=W
$$




$$
V_{T}=\sqrt{\frac{2 W}{C_{D} \rho S}}
$$

A computational fluid dynamic analysis was performed on a cluster of supercomputers located at the United States Air Force Academy CFD Lab running a Cobalt flow solver. Cobalt solves the laminar and turbulent NavierStokes equations for unsteady, compressible cases. Turbulence and detached flow modeling were achieved with the Detached-Eddy Simulation, which solves large eddies in the flow field and models smaller eddies. ${ }^{1}$ This method was refined by several professors at the United States Air Force Academy. The Reynolds-Averaged Navier Stokes method was used to solve regions of attached flow. The grid used for the study was fabricated by an outside contractor due to the highly complex nature of the ARGUS design. ${ }^{2}$

\section{Uncertainty}

An uncertainty analysis using the AIAA total-systems approach was performed for all testing. ${ }^{3}$ Both bias error and precision error contributed to the overall uncertainty. A root-sum-square method was used to determine the overall uncertainty, as shown in Eq. 2, where $\mathrm{U}_{\mathrm{i}}$ is the overall uncertainty, $\mathrm{B}_{\mathrm{i}}$ is the bias error the measurements, and $\mathrm{P}_{\mathrm{i}}$ is the precision error the measurements. ${ }^{4}$

$$
U_{i}=\sqrt{B_{i}^{2}+P_{i}^{2}}
$$

To minimize bias error, the test equipment was calibrated to the highest standards possible before each phase of testing. During this calibration, bias error influence coefficients were determined for each specific piece of test equipment. The ABLE force balance had 10 total bias error coefficients, 1 for the positive and negative direction of each measured force shown in Fig. 3. The calibration of the test equipment reduced the contribution of bias error to less than $10 \%$ of the overall error. The precision error, which is a function of the standard deviation of the 200 data samples taken at each data point (taken at 100 $\mathrm{Hz}$ for 2 seconds), was much more difficult to minimize. This was due primarily to oscillations that were apparent in the time history data recorded during Phase III testing. These oscillations resulted from low-amplitude vibration of the ARGUS model and increased the standard deviation of the data samples collected during the 2-second intervals, which thereby increased the precision error and thus the overall uncertainty of the data during all three Phases of testing. The yawing moment coefficient had the greatest percent uncertainty, mainly because the yawing moments experienced by the ARGUS were of very small magnitude, especially in comparison to the pitching moment coefficient. This small magnitude resulted in a large percentage of uncertainty in the results, even with small absolute values of uncertainty. However, because the time-history data

Table 2. Average Uncertainty Calculated ARGUS Coefficients.

\begin{tabular}{|l|l|l|l|l|}
\hline Mach & $\mathbf{C}_{\mathbf{L}}$ & $\mathbf{C}_{\mathbf{N}}$ & $\mathbf{C}_{\mathbf{M}}$ & $\mathbf{C}_{\mathbf{D}}$ \\
\hline 0.2 & $\pm 14 \%$ & $\pm 81 \%$ & $\pm 1 \%$ & $\pm 2 \%$ \\
\hline 0.5 & $\pm 16 \%$ & $\pm 78 \%$ & $\pm 2 \%$ & $\pm 1 \%$ \\
\hline
\end{tabular}

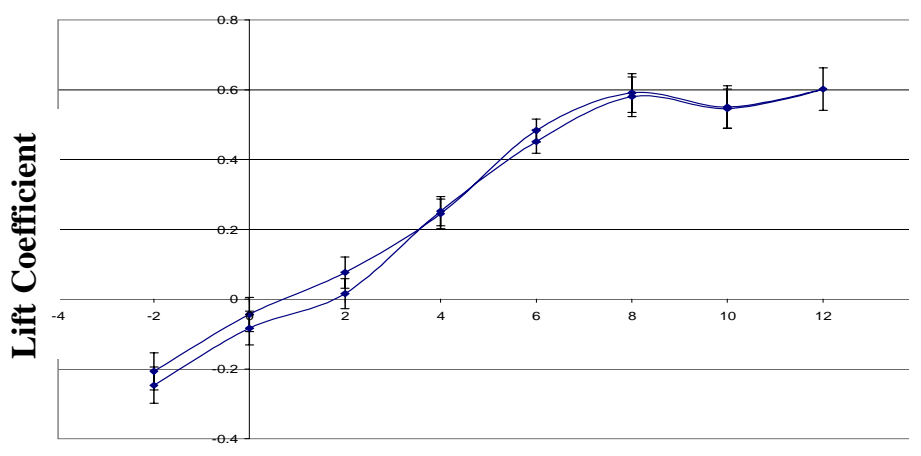

Angle of Attack, deg

\section{Figure 5. Phase III ARGUS Design Lift Coefficient, Mach 0.2, With Uncertainty Error Bars Overlaid.} showed that the large standard deviations in the data were from in oscillations around the average value reported in testing, the uncertainty calculated for this testing can be considered worst case. Fig. 5 presents the lift coefficient for the Phase III Argus design with appropriate error bars. It can be seen that, even with worst-case uncertainty, valid data comparisons can be made. Table 2 presents the average uncertainty for each calculated coefficient through all Phases of testing. 


\section{Experimental Results}

\section{A. Phase I: Fall 2003}

Phase I testing established baseline aerodynamic data for the initial ARGUS design, which is presented in Fig. 1 and Fig. 6, and which included solid drag brakes. Results from this initial testing showed that ARGUS had undesirable aerodynamic characteristics in several areas. First, ARGUS exhibited a negative lift-curve slope, especially at low angles of attack. This can be seen in Fig. 7, with the baseline design (or with the solid drag brakes). As discussed earlier, this negative lift-curve slope could potentially cause the ARGUS to rise towards the carrier aircraft upon release. Yawing moment excursions were found to be large with variation in angle of attack, as seen in Fig 8. Additionally, the data showed that ARGUS had near-neutral longitudinal stability near the trim angle of attack of $0^{\circ}$, as seen in Fig 9. Fig. 10 presents the drag

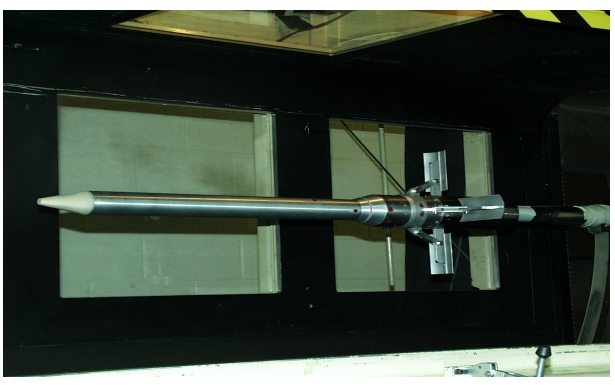

Figure 6. Phase I ARGUS Design On Test Sting.

coefficients obtained for the Phase I ARGUS design. In addition to the yawing moment characteristics, the near-

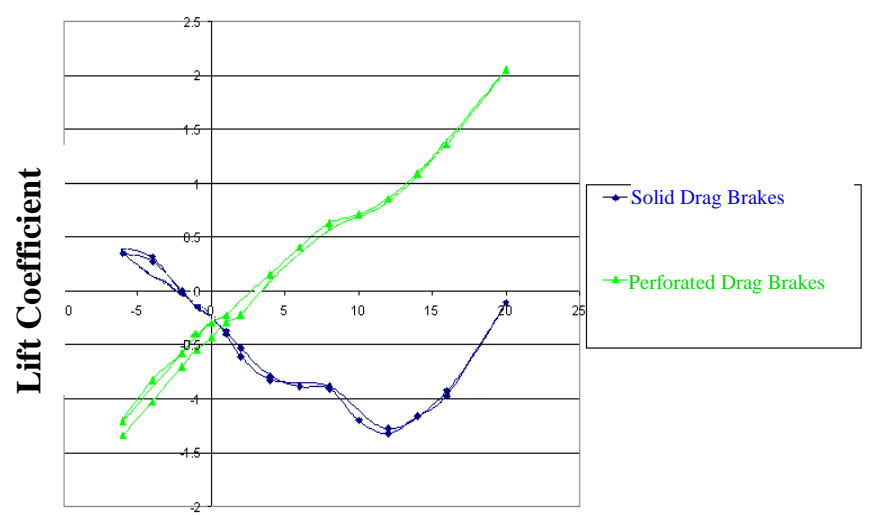

Angle of Attack, deg

Figure 7. Lift Coefficient As a Function Of Angle-of-Attack For Phase I ARGUS Design.

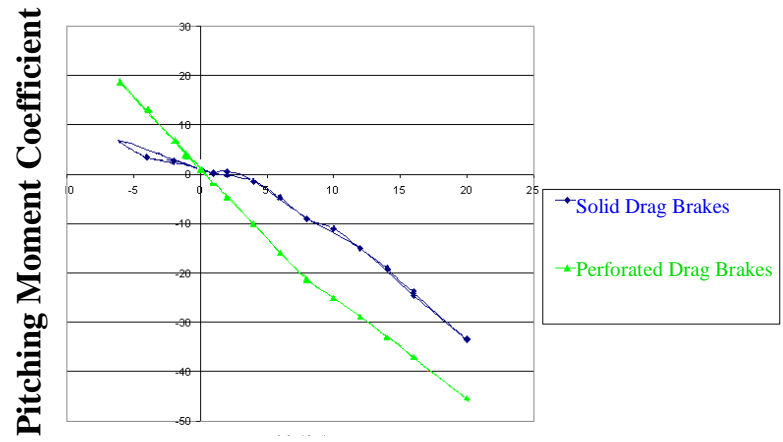

Angle of Attack, deg

Figure 9. Pitching Moment Coefficient As a Function Of Angle-of-Attack For Phase I ARGUS Design.

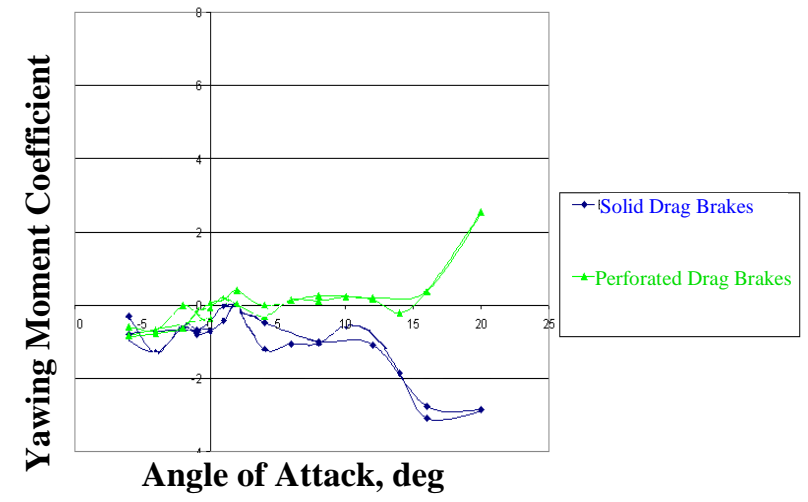

Figure 8. Yawing Moment Coefficient As a Function Of Angle-of-Attack For Phase I ARGUS Design.

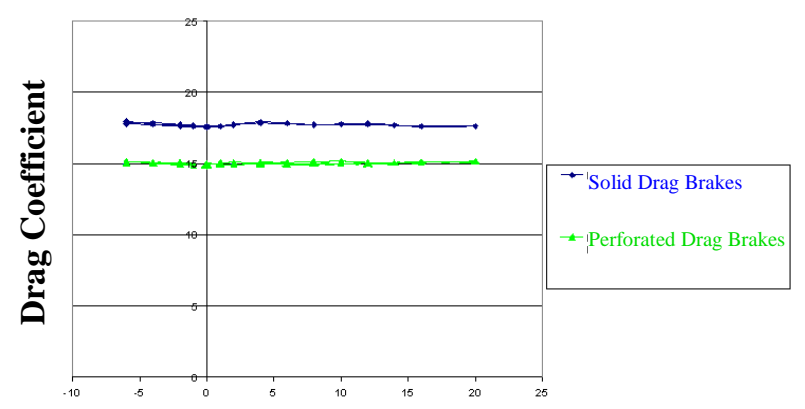

Angle of Attack, deg

Figure 10. Drag Coefficient As a Function Of Angle-of-Attack For Phase I ARGUS Design. 


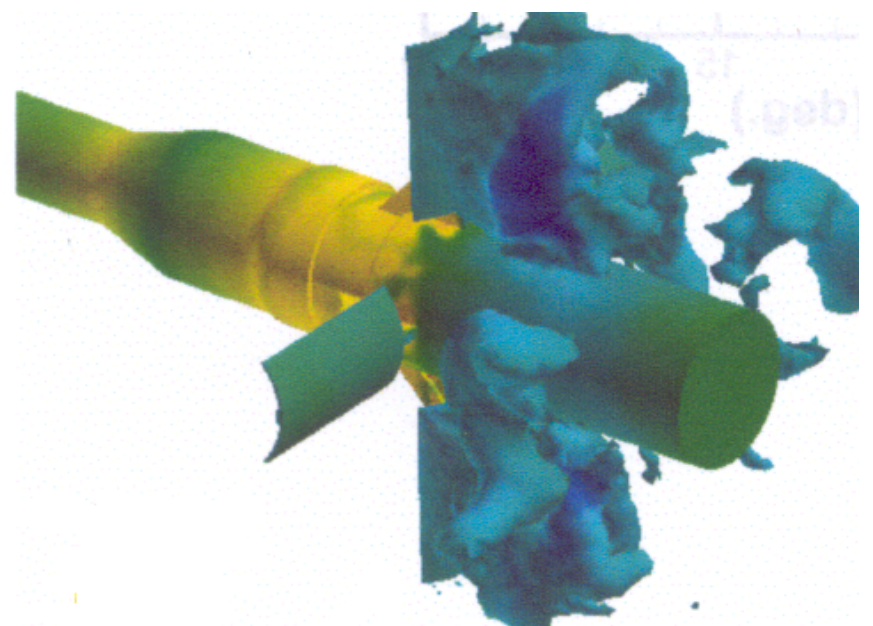

Figure 11. CFD Simulation Image Showing Flow Field Around Aft Section of the ARGUS. neutral longitudinal stability was a probable cause of the coning experienced in flight tests. It was concluded that the flow interaction between the drag brakes and the aft body of the ARGUS caused these adverse aerodynamic characteristics. Specifically, there was likely asymmetric vortex shedding occurring off of the drag brakes that was impacting the aft section of the main body, causing poor lift and longitudinal stability characteristics, as well as yawing moment excursions. This hypothesis was affirmed by CFD results, presented in Fig. 11, which shows strong vortex shedding off the solid drag brakes. ${ }^{2}$

It can be seen in Fig. 7 that the ARGUS generated negative lift at $0^{\circ}$ angle-of-attack, while in Fig. 9 it can be seen that a positive (nose-up) pitching moment was also present. These traits, which were apparent in all phases of testing, were attributed to a slight nose-down attitude of the

ARGUS model while mounted on the test sting at $0^{\circ}$ angle-of-attack. Since the same forebody was used during all phases of testing and only increased in size as necessary, a slight flaw in the original fabrication likely caused this abnormality.

Additional efforts during Phase I were aimed at mitigating the adverse aerodynamic characteristics seen in initial testing. These approaches included: 1) using "blocker plates" to remove the space between the drag brakes and the ARGUS main body, as seen in Fig. 12, and 2) adding perforations to the drag brakes (without the blocker plates). ${ }^{5}$ It was established in previous testing that the ARGUS had desirable aerodynamic characteristics without the drag brakes deployed, so these two methods were used in an attempt to correct the problems that became apparent with the addition of the drag brakes. The main ARGUS body was not changed throughout Phase I testing. The attempts to mitigate these adverse characteristics yielded very different results.

The addition of the blocker plates, which eliminated the airflow in the

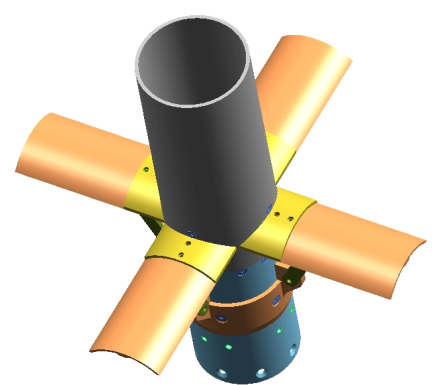

Figure 12. ARGUS Aft Body With Blocker Plates Installed Below Drag Brakes. gap between the drag brakes and the aft body, worsened all of the negative trends observed before this modification. Fig. 13 shows that the lift curve slope was even more negative with the blocker plates, while Fig. 14 shows that neutral longitudinal stability or instability was exhibited near $0^{\circ}$ angle-of-attack.

The greatest improvements in the aerodynamic characteristics were obtained from adding perforations to the

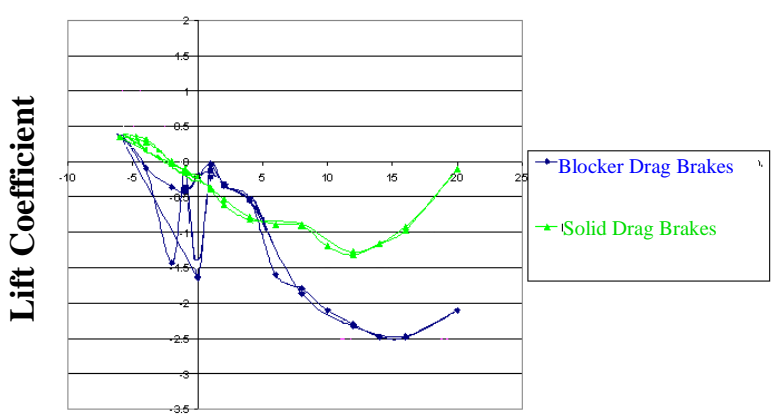

Angle of Attack, deg

Figure 13. Lift Coefficient As a Function Of Angle-of-Attack For Phase I ARGUS Design.

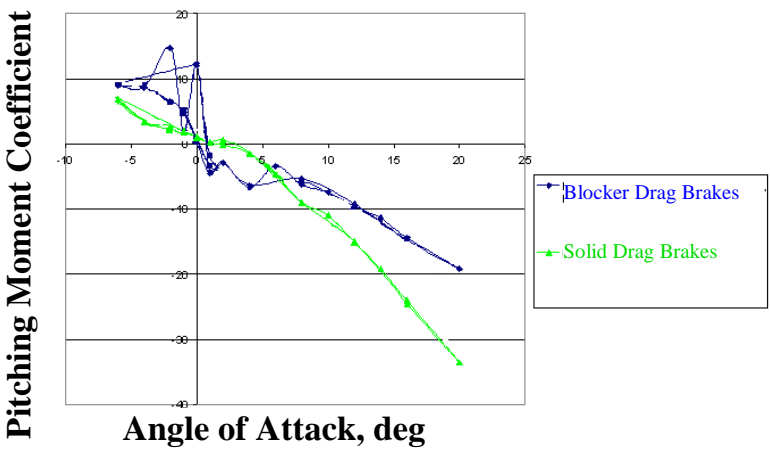

Figure 14. Pitching Moment Coefficient As a Function Of Angle-of-Attack For Phase I ARGUS Design.

6

American Institute of Aeronautics and Astronautics 
drag brakes, as can be seen in the comparisons in Fig 7, Fig. 8, and Fig 9. Adding perforations created a nearly linear positive lift-curve slope, gave very stable longitudinal stability about the trim angle of attack of $0^{\circ}$, and reduced the magnitude of the yawing moment excursions.

The goal for terminal velocity of the ARGUS was initially set at $265 \mathrm{ft} / \mathrm{sec}$. From Fig. (10), the drag coefficient at $0^{\circ}$ angle-of-attack can be seen to be approximately 15 for the perforated design and approximately 17.5 for the solid drag-brake design. The weight of the ARGUS was assumed to be $65 \mathrm{lbs}$, the design point during this Phase in testing. Using the method in Eqs. (1), the perforated drag brakes were found to have a terminal velocity of 260 $\mathrm{ft} / \mathrm{sec}$, while the increased drag of the solid drag brakes lowered the terminal velocity to $230 \mathrm{ft} / \mathrm{sec}$. Therefore, in addition to the gains described above, perforating the drag brakes also allowed for the ARGUS to achieve a terminal velocity closer to the prescribed goal. Therefore, at the end of Phase I testing, the ARGUS was found to have suitable aerodynamic characteristics in all areas of interest.

\section{B. Phase II: Spring 2004}

It was decided after examination of the results of Phase I testing to incorporate drag brake perforations into the working ARGUS design to mitigate the asymmetric vortex shedding from the drag brakes. The focus of the Phase II testing was to optimize the perforation pattern of the drag brakes. The drag brake perforation pattern used in Phase I was defined as the baseline design and variations were made to the size of the holes and their alignment in an attempt to further

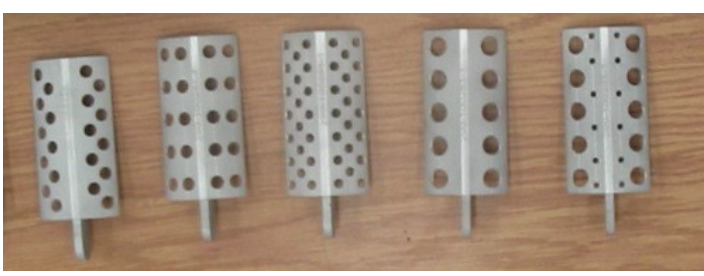

Figure 15. Baseline, Aligned, Small, Large, and Mixed Perforation Configurations.

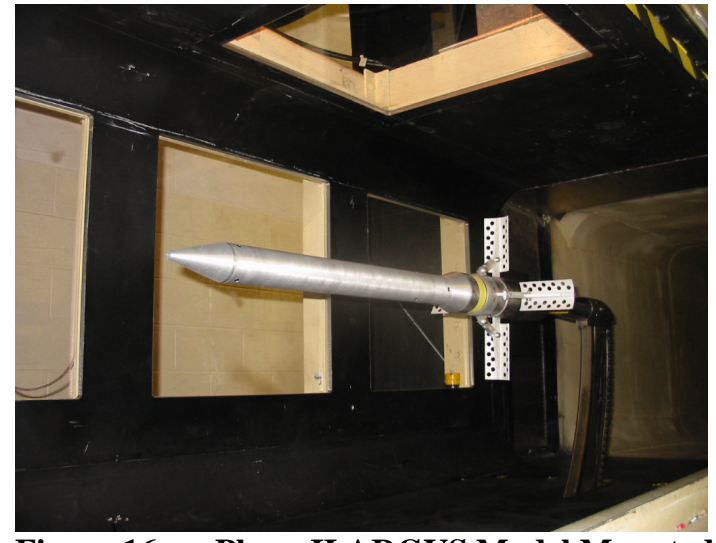

Figure 16. Phase II ARGUS Model Mounted in Wind Tunnel.

improve the aerodynamic characteristics of the ARGUS. Additionally, a "mixed" configuration of large and small perforations was also tested. The five drag brake configurations evaluated are presented in Fig 15. This investigation was one of the few documented cases where the effects of perforation patterns on drag-inducing devices were examined. Though the perforation configurations were varied, the ratio of the area of the holes to the area of the drag brake was kept constant throughout testing. Additionally, the weight increased to $80 \mathrm{lbs}$ and the size of the forebody was increased to 3.5 " from 3.25" (full-scale) to accommodate internal component growth, so the Phase II testing also provided baseline data for the new, larger ARGUS design. Fig. 16 shows the Phase II ARGUS design with the baseline drag brakes mounted on the test sting in the wind tunnel.

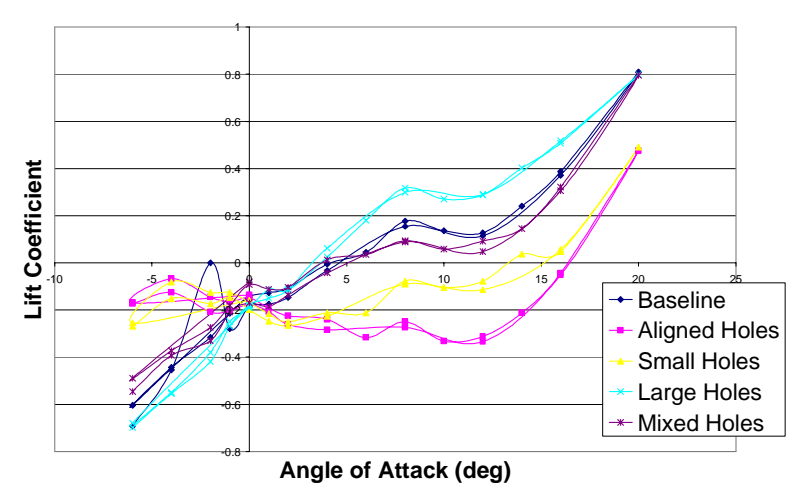

Figure 17. ARGUS Lift Coefficient Versus Angle of Attack for Various Perforation Designs.

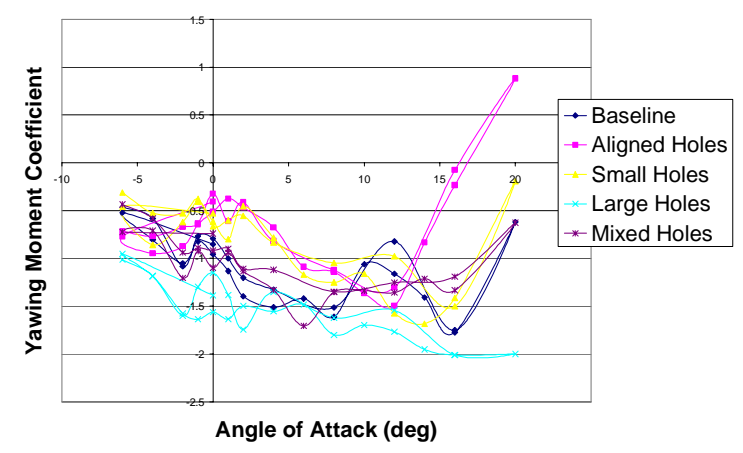

Figure 18. ARGUS Yawing Moment Coefficient Versus Angle of Attack for Various Perforation Designs. 


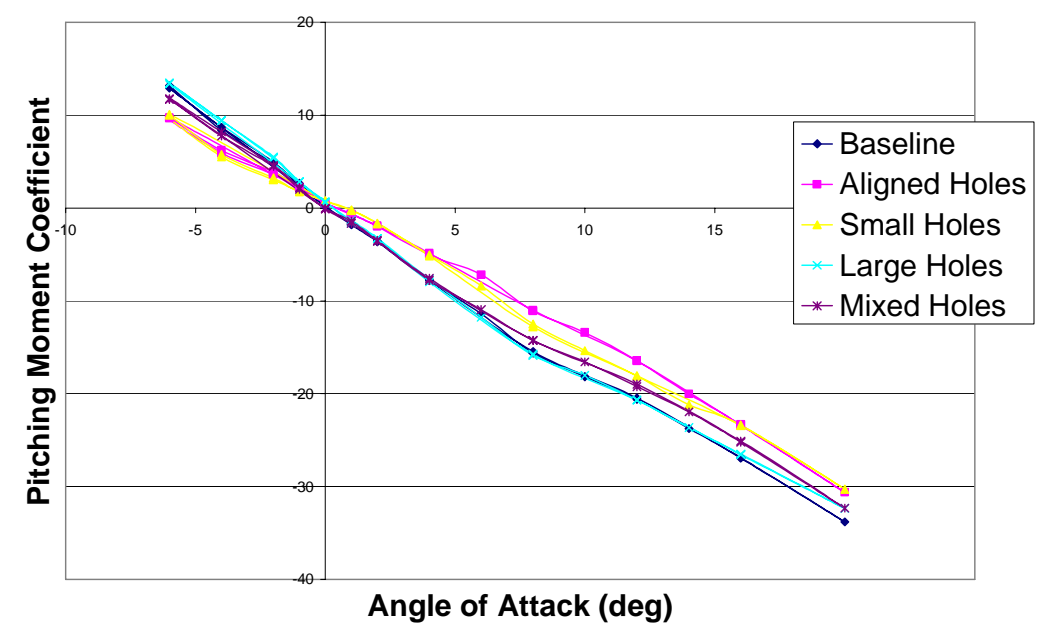

Figure 19. ARGUS Pitching Moment Coefficient Versus Angle of Attack for Various Perforation Designs.

that grew in intensity with increasing angle of attack. The baseline and mixed configuration again produced similar results, while the aligned and small holes, providing the least desirable lift characteristics, interestingly exhibited the smallest yawing moment excursions. Fig. 19 shows that all drag brake configurations exhibited longitudinal static stability, with the baseline configuration demonstrating the most stability, and the aligned configuration the worst; however, all of the configurations exhibited acceptable pitching moment characteristics. The drag coefficient for each configuration is presented in Fig. 20. Using Eqn. 1, the baseline configuration was found to have the lowest terminal velocity of $256 \mathrm{ft} / \mathrm{sec}$, and the mixed configuration was the highest at $260 \mathrm{ft} / \mathrm{sec}$. The slight variation in the terminal velocity between the drag brake perforation designs was attributed to the fact that only the perforation pattern was changed on the drag brakes, while the hole/area ratio was kep

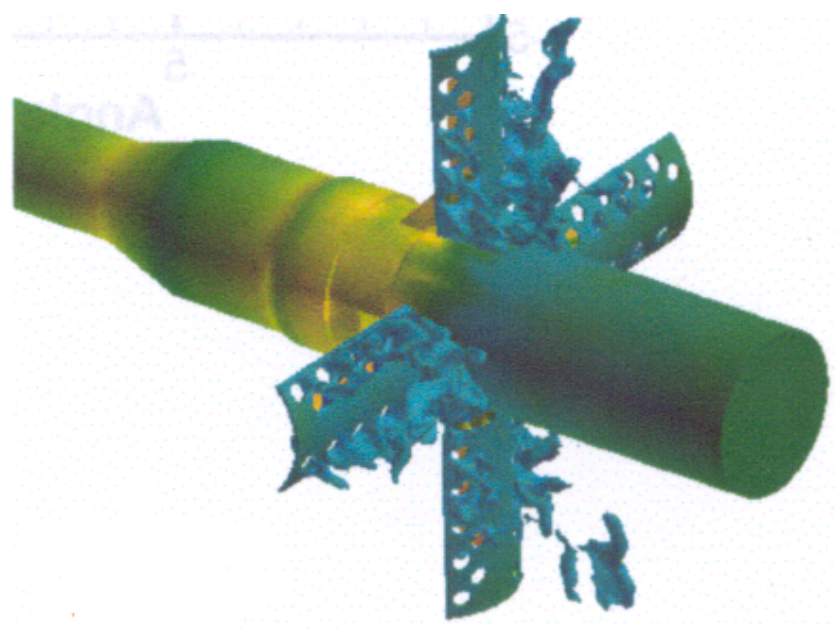

Figure 21. CFD Simulation Image Showing Flow Field Around Aft Section of the Phase II ARGUS Design.
Fig 17 shows that the lift characteristics of the baseline and mixed configuration perforated drag brake designs were comparable and did not exhibit any major undesirable characteristics. The large-hole configuration provided a steeper lift-curve slope and therefore more desirable lift characteristics, while the aligned holes and small holes exhibited undesirable lift curves. Despite the fact that the large holes provided the most desirable lift characteristics, Fig. 18 shows that the large holes also provided the least desirable yawing moment characteristics, in that it exhibited large yawing moment excursions

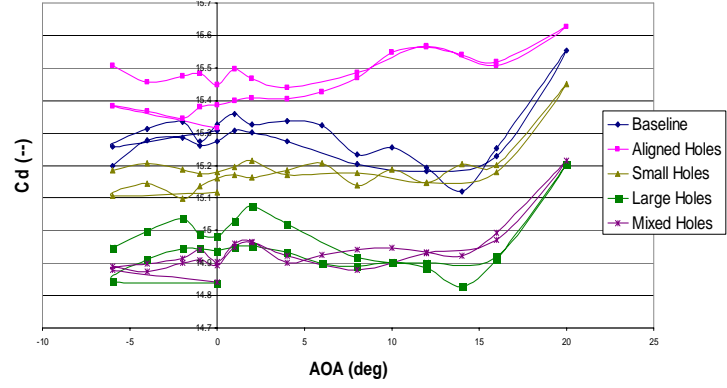

Figure 20. ARGUS Drag Coefficient Versus Angle of Attack for Various Perforation Designs. (note: decreased scale for ease of differentiation)
With the Phase II ARGUS configuration and perforated drag brake designs, the terminal velocity projections were close to the desired target terminal velocity. This was due to an effort by Textron, Inc. to match the size and drag of the new drag brakes to the larger, heavier ARGUS design. ${ }^{6} \quad$ The positive effect of the drag brake perforations can also be seen in Fig. 21, which is an image of the CFD analysis done on the ARGUS with the perforations added to the drag brakes at Mach 0.2 and $6^{\circ}$ angle of attack. ${ }^{2}$ Comparing Fig. 11 with Fig 21, it can be seen that the addition of the perforations mitigated the large vortices that were occurring behind the drag brakes of the ARGUS, allowing for improved aerodynamic performance.

This testing was able to quantify the effects of the differing perforation patterns on the aerodynamic characteristics of the ARGUS. As a result of the testing, either the baseline or the 
mixed perforations were found to be suitable for the ARGUS design. The baseline perforation design was chosen based on producibility considerations and successful drop tests with this configuration, which occurred concurrently with the Phase II testing. This baseline configuration produced suitable aerodynamic characteristics in all areas of interest.

\section{Phase III: Fall 2004 Testing}

The ARGUS design had gone through many changes during the summer of 2004 and was beginning to focus on a final design prior to beginning the Phase III testing. A design comparison between the three Phases of testing is provided in Table (3). In Phase III, the length of the fullscale design was increased 2 inches in the aft section to allow room for all the necessary electronics. Furthermore, the drag brakes were located 1 inch further aft on the tail can of ARGUS. This increased size and slightly different configuration necessitated further wind-tunnel research, as vortex interaction with the aft section was one of the root caus plagued the design in its early stages. Also, attention was fo

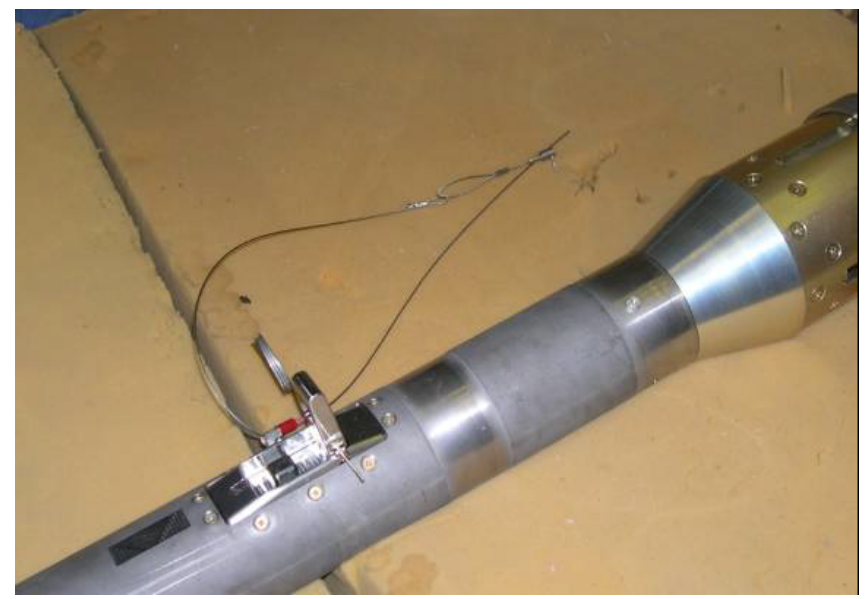

Figure 22. Lanyard Release System On Phase III ARGUS Model

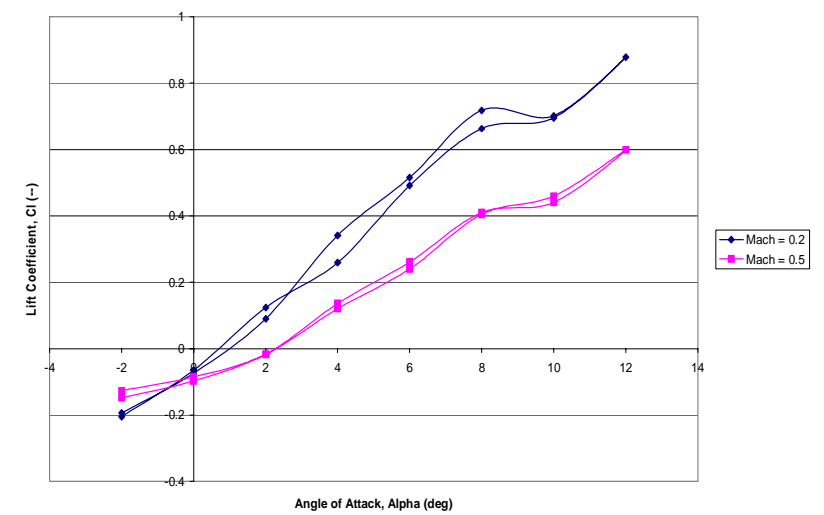

Figure 23. Phase III ARGUS Lift Coefficient, Mach 0.2 and 0.5 .
Table 3. Phase I, II, and III ARGUS Design Dimensions.

\begin{tabular}{|c|c|c|c|c}
\hline $\begin{array}{c}\text { Testing } \\
\text { Period }\end{array}$ & Length & Weight & $\begin{array}{c}\text { Drag } \\
\text { Brake } \\
\text { Design }\end{array}$ & $\begin{array}{c}\text { Forebody } \\
\text { Diameter }\end{array}$ \\
\hline Phase I & 65 in. & $65 \mathrm{lbs}$ & Solid & 3.25 in. \\
\hline Phase II & 65 in. & $\mathbf{8 0} \mathrm{lbs}$ & Perforated & 3.5 in. \\
\hline Phase III & 67 in. & $\mathbf{8 0} \mathrm{lbs}$ & Perforated & 3.5 in. \\
\hline
\end{tabular}

causes of the adverse aerodynamic characteristics that ocused on achieving the desired terminal velocity of approximately $265 \mathrm{ft} / \mathrm{sec}$.

There were three objectives to the Phase III ARGUS testing. The first objective was to define the baseline aerodynamic characteristics for the new ARGUS design. In addition, it was determined that a lanyard system, shown in Fig. 22, would be used to stow the drag brakes during carriage aboard an aircraft, and then deploy the drag brakes upon release. This lanyard system would remain with the ARGUS during its descent. Therefore, the second objective was to evaluate the inclusion of the lanyard system on the ARGUS to ensure that it would not create any aerodynamic problems during flight. Additionally, this testing was accomplished using not only the time-averaged data that had been analyzed in previous tests, but also with time history data. Thus, the third objective was to identify any unsteady phenomena not seen with the timeaveraged method in Phase I and II.

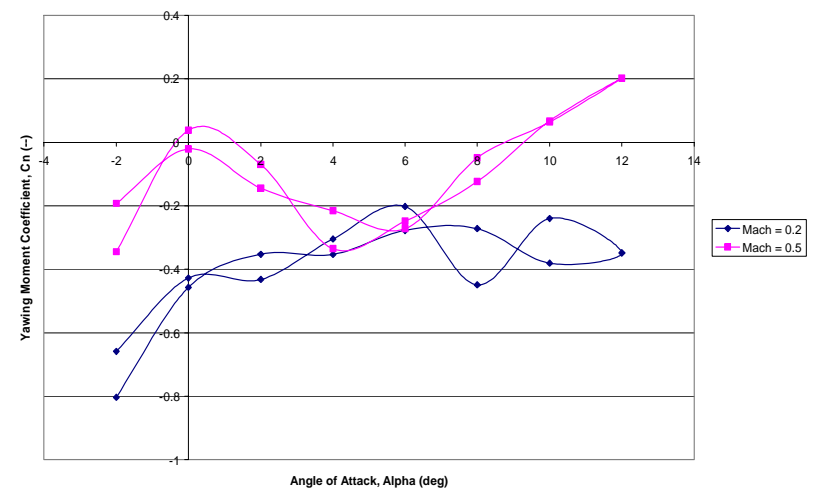

Figure 24. Phase III ARGUS Yawing Moment Coefficient, Mach 0.2 and 0.5. 


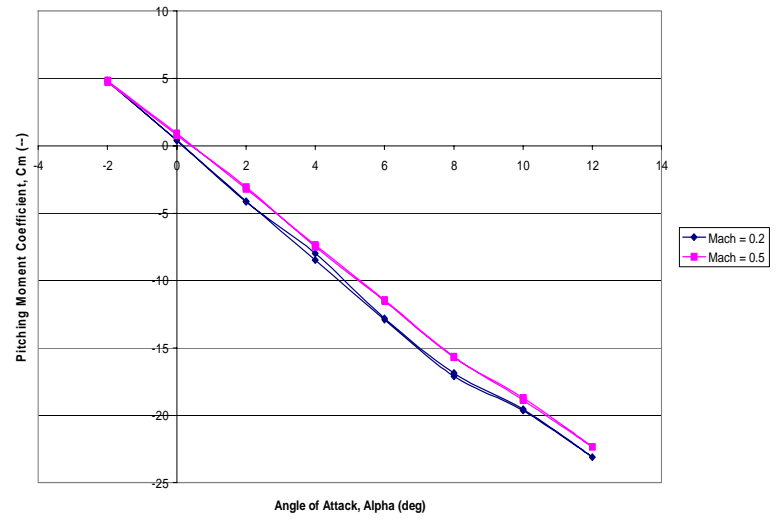

Figure 25. Phase III ARGUS Pitching Moment Coefficient, Mach 0.2 and 0.5.

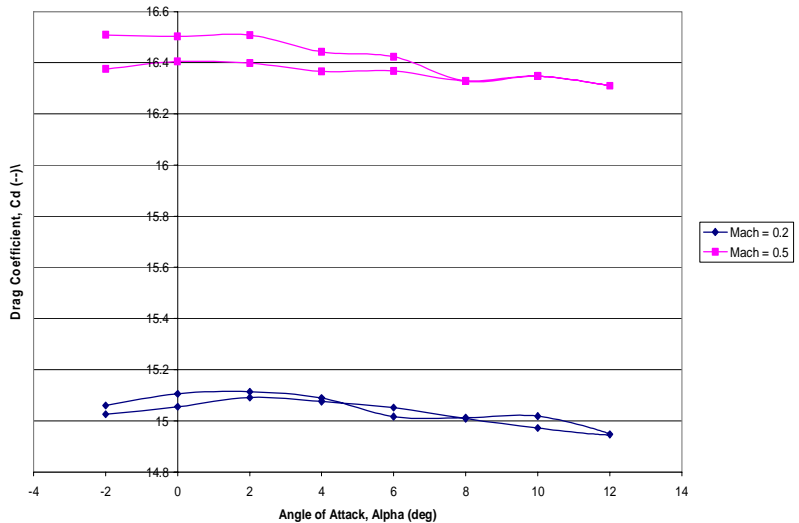

Figure 26. Phase III ARGUS Drag Coefficient, Mach 0.2 and 0.5.

Testing revealed that the addition of the lanyard and the larger aft section did not degrade any of the aerodynamic characteristics seen in previous testing. Fig. 23, Fig. 24, Fig. 25 and Fig. 26 present the results from the four aerodynamic areas of focus. Prior to this, results have only been presented at Mach 0.2, as results at higher Mach numbers closely mirrored the results from Mach 0.2. However, a Mach comparison is presented in Figs. 23, 24, 25, and 26 for Phase III that is representative of the Mach effects apparent in all Phases of testing. Fig. 23 shows that increased Mach number decreased the lift-curve slope; however, the lift characteristics of the ARGUS were not objectionable at any Mach number tested. Fig. 24 shows that increased Mach number created yawing moment excursions that were greater than at Mach 0.2, however, the larger excursions were not of sufficient magnitude to constitute objectionable characteristics. Fig. 25 shows that the pitching moment exhibited slightly less longitudinal static stability with increased Mach number, but again this decreased stability was not considered objectionable. The drag coefficients, presented in Fig. 26, show that the ARGUS drag coefficients increased at higher Mach numbers; however, it should be pointed out that the drag coefficient at Mach 0.2 was the value used in the calculation of the terminal velocity. The terminal velocity for the Phase III ARGUS design was calculated to be $258 \mathrm{ft} / \mathrm{sec}$. This small increase in terminal velocity over Phase II with the baseline perforated drag brakes was attributed to the one inch further aft location of the drag brakes on the aft body.

A time-history investigation of the Phase III test data showed that oscillations were apparent in all of the coefficients calculated, as shown in Fig 27. These oscillations were at approximately the same frequency for the drag, pitching moment, side force, and yawing moment coefficients, and at approximately twice that

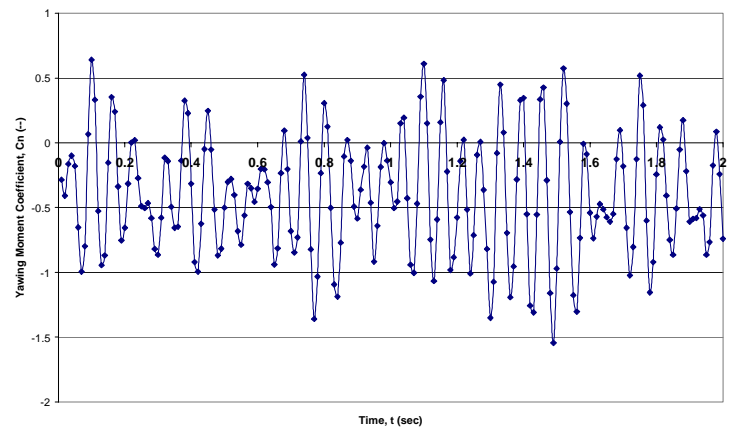

Figure 27. Time History of Phase III ARGUS Yawing Moment Coefficient, Mach 0.2, $8^{\circ}$ a, 2Second Sample at $100 \mathrm{~Hz}$

frequency for the lift coefficient. These oscillations are likely a result of the ARGUS model support configuration, composed of the ARGUS model, force balance, and the test sting. These oscillations did not appear to increase or decrease in magnitude for the two-second period over which the data was collected.

As a result of Phase III testing, the ARGUS was now found to have acceptable aerodynamic characteristics in all areas of interest.

\section{Conclusions}

Wind tunnel and CFD efforts at the United States Air Force Academy were essential to development of the final ARGUS design. There were three major conclusions reached during research. During Phase I, perforated drag brakes significantly improved the aerodynamic stability by mitigating the effects of asymmetric vortex shedding. During Phase II, the baseline and mixed drag brake designs created optimum aerodynamic characteristics. During 
Phase III, the addition of the lanyard system did not degrade the overall aerodynamic characteristics of the ARGUS, and time history data showed that constant-frequency oscillations occurred during testing but did not provide performance concerns. As a result of increased weight and a shift in the location of the drag brakes, the Phase III configuration was projected to produce a terminal velocity close to the target, thus fulfilling all aerodynamic requirements for the ARGUS.

\section{Recommendations}

As a result of the testing, two recommendations were made. First, additional flow visualization methods or CFD analysis is recommended to better understand the complex flow occurring behind the drag brakes that were the primary cause of the initial adverse aerodynamic characteristics. Second, flight validation of the final ARGUS design with the lanyard system attached is recommended to ensure that the final design is suitable for the ARGUS mission.

\section{Acknowledgments}

The authors wish to thank the United States Air Force Academy Department of Aeronautics for their support of this wind-tunnel investigation. This support included the use of test facilities, supercomputers, and machine fabrication shops. In addition, Lt Colonel David Wetlesen of the Department of Aeronautics was instrumental in serving as overall program coordinator. Also, Textron, Inc. was invaluable in providing the overall design lead for ARGUS configurations, fabrication of the model, and guidance on specific test emphasis items.

\section{References}

${ }^{1}$ Forsythe, James R., Hoffmann, Klaus A., Cummings, Russell M., and Squires, Kyle D., "Detached-Eddy Simulations With Compressibility Corrections Applied to a Supersonic Axisymmetric Base Flow," Journal of Fluids Engineering, Vol. 124, No. 4, 2002, pp. 911-923.

${ }^{2}$ Cummings, Russell with Wetlesen, David. "A CFD Analysis for the Advanced Remote Ground Unattended Sensor (ARGUS) Configuration,”USAFA DFAN TR 04-02.

3“Assessment of Experimental Uncertainty with Application to Wind-Tunnel Testing," AIAA Standard S-071A-1999.

${ }^{4}$ Schneider, Joshua, Searcy, Jeffrey, and Yechout, Thomas, "Investigation and Optimization of NASA Orbital Space Plane Designs," USAFA DFAN TR 04-03.

${ }^{5}$ Guthman, Caleb et al., "A Wind Tunnel Evaluation of Advanced Remote Ground Unattended Sensor (ARGUS) Configurations," USAFA DFAN TR 04-01.

${ }^{6}$ Florendo, Corey, Gilbert, Jonathon, and Yechout, Thomas, "A Wind Tunnel Evaluation of Perforated Drag Brake Designs for the Advanced Remote Ground Unattended Sensor (ARGUS)," USAFA DFAN TR 04-04. 